\title{
Theory of Spin Hall Effect
}

\section{in Random $\mathrm{Pt}-\mathrm{M}(\mathrm{M}=\mathrm{V}, \mathrm{Nb}, \mathrm{Ta})$ Alloys}

\author{
I. TUREK ${ }^{a, *}$, J. KUdRNOVSKÝ ${ }^{b}$ AND V. DRCHAL ${ }^{b}$ \\ ${ }^{a}$ Institute of Physics of Materials, Czech Academy of Sciences, Žižkova 22, 61662 Brno, Czech Republic \\ ${ }^{b}$ Institute of Physics, Czech Academy of Sciences, Na Slovance 2, 18221 Praha, Czech Republic
}

\begin{abstract}
The spin Hall effect in fcc Pt-rich disordered solid solutions Pt-M ( $\mathrm{M}=\mathrm{V}, \mathrm{Nb}$, Ta) is studied by means of a relativistic $a b$ initio Green-function theory. We find that the spin Hall angle of all three alloy systems exhibits similar sizable values, with a maximum slightly above 0.2 , obtained for dopant concentrations around 12 at.\%. This result is explained by a competition between opposite concentration trends of the intrinsic spin Hall conductivity and of the longitudinal residual resistivity while the strength of the impurity spin-orbit interaction plays only a minor role. This behavior agrees qualitatively with results of recent experiments on spin Hall torques in multilayered systems involving Pt-rich alloys with heavy and light impurities.
\end{abstract}

DOI: 10.12693/APhysPolA.137.717

PACS/topics: spin Hall effect, ab initio theory, random alloys

\section{Introduction}

The spin Hall effect represents one of the most important phenomena in the field of spintronics [1]. This spinorbit induced transverse transport phenomenon thus attracts ongoing attention motivated by the need for efficient sources of spin currents and spin torques in various magnetoelectronic devices. The relevant physical property, namely, the spin Hall conductivity (SHC) has been addressed in a number of experimental and theoretical studies. Its relation to the longitudinal electrical conductivity defines the so-called spin Hall angle (SHA), which is a dimensionless parameter that controls the efficiency of charge to spin conversion.

The search for materials with large SHA values has traditionally been focused on $5 d$ transition metals and other heavy elements, including their mutual diluted and concentrated alloys, see e.g., [1-3] and references therein. However, a recent experimental study involving Pt-rich random $\mathrm{Pt}-\mathrm{Hf}$ and $\mathrm{Pt}-\mathrm{Al}$ alloys has revealed a similar enhancement of the spin Hall torques in multilayers based on these alloy systems [4]. This finding contradicts a generally accepted role of strong spin-orbit interaction of the impurity atoms as a necessary prerequisite for large SHC and SHA values of the alloy. Since this aspect of the spin Hall effect has not been studied systematically, the present work is devoted to a relativistic ab initio theory of transport properties of fcc random concentrated $\mathrm{Pt}-\mathrm{M}$ alloys, where the dopant $\mathrm{M}$ runs over three isoelectronic transition-metal elements, $\mathrm{M}=\mathrm{V}, \mathrm{Nb}$, and $\mathrm{Ta}$, which mutually differ mainly by the strength of their spin-orbit coupling. This choice of alloying elements $\mathrm{M}$ is partly motivated by the large spin Hall effect encountered in Ta-based systems $[2,3]$.

\footnotetext{
*corresponding author; e-mail: turek@ipm.cz
}

\section{Methods and numerical details}

The electronic structure of the studied disordered alloys was obtained in the all-electron self-consistent relativistic tight-binding linear muffin-tin orbital (TB-LMTO) method and the coherent potential approximation (CPA) [5]. The valence basis consists of $s^{-}$, $p$-, and $d$-type orbitals and the one-electron potentials and electron densities were treated in the atomic sphere approximation with the Wigner-Seitz radii set according to the Vegard law using the experimental Wigner-Seitz radii of pure metals $\mathrm{Pt}, \mathrm{V}, \mathrm{Nb}$, and Ta.

The transport properties were studied by means of the Kubo linear response theory modified in the relativistic TB-LMTO-CPA technique [6]. This approach yields the ordinary conductivity tensor $\sigma_{\mu \nu}$, where $\mu, \nu \in\{x, y, z\}$, which provides the residual conductivity and resistivity of random alloys. For studies of the SHC, one has to introduce the spin-dependent conductivity tensor $\sigma_{\mu \nu}^{\lambda}$, where the superscript $\lambda \in\{x, y, z\}$ refers to the spin polarization of the electric current flowing along $\mu$ axis. The tensor $\sigma_{\mu \nu}^{\lambda}$ at zero temperature is then defined by:

$$
\begin{gathered}
\sigma_{\mu \nu}^{\lambda}=-2 \sigma_{0} \int_{-\infty}^{E_{\mathrm{F}}} \operatorname{Tr}\left\langle\left(\sigma^{\lambda} v_{\mu}\right) g_{+}^{\prime}(E) v_{\nu}\left[g_{+}(E)-g_{-}(E)\right]\right. \\
\left.-\left(\sigma^{\lambda} v_{\mu}\right)\left[g_{+}(E)-g_{-}(E)\right] v_{\nu} g_{-}^{\prime}(E)\right\rangle \mathrm{d} E
\end{gathered}
$$

which describes the linear response of the spin current $\tilde{v}_{\mu}^{\lambda}=\sigma^{\lambda} v_{\mu}$ to a spin-independent electrical field in direction of $\nu$ axis. Next, the quantity $\sigma_{0}$ is a prefactor comprising basic physical constants and the crystal volume, $E_{\mathrm{F}}$ denotes the Fermi energy, $E$ is a real energy variable, the trace $(\operatorname{Tr})$ is taken over all valence orbitals of the crystal, the quantities $\sigma^{\lambda}$ are the Pauli spin matrices, the quantities $v_{\mu}$ and $v_{\nu}$ represent the velocity (current) operators, $g_{ \pm}(E)$ denote the retarded and advanced Green functions, the prime at $g_{ \pm}(E)$ denotes 
energy derivative, and the brackets $\langle\ldots\rangle$ refer to the configuration averaging for random alloys.

The configuration average in Eq. (1) was done in close analogy to the case of the ordinary conductivity tensor $\sigma_{\mu \nu}$, developed within the CPA in $[6,7]$. As a result, the final value of $\sigma_{\mu \nu}^{\lambda}$ in (1) is given as a sum of the Fermisurface and Fermi-sea terms. Both terms contain a coherent contribution (corresponding to a replacement of $\langle g g\rangle$ by $\langle g\rangle\langle g\rangle$ in the CPA average) and an incoherent contribution (equal to the CPA-vertex corrections). The sum of both coherent contributions can be identified with the intrinsic part of the tensor $\sigma_{\mu \nu}^{\lambda}$ while the sum of both incoherent contributions defines its extrinsic part. More details will be given elsewhere.

The numerical implementation was done similarly to recent calculations of the Fermi-surface [7] and Fermisea [6] terms of the conductivity tensor $\sigma_{\mu \nu}$. In particular, a very fine sampling of the Brillouin zone, employing $\approx 10^{8}$ mesh points, was used for the Fermi-surface term while the Fermi-sea term was reformulated as an integral over a complex-energy contour, which was evaluated by using 28 complex nodes distributed along the contour.

Since the systems studied in this work possess full cubic symmetry, the only non-vanishing independent element of the tensor $\sigma_{\mu \nu}^{\lambda}(1)$ is $\sigma_{x y}^{z}$. The SHC discussed below was thus identified with this element. The conductivity tensor $\sigma_{\mu \nu}$ reduces to a single non-vanishing independent element, as well as denoted by $\sigma_{x x}$. Its reciprocal value defines the longitudinal residual resistivity $\rho$, i.e., $\rho=1 / \sigma_{x x}$. Finally, the SHA is obtained as $\sigma_{x y}^{z} / \sigma_{x x}=\sigma_{x y}^{z} \rho$.

\section{Results and discussion}

According to assessed equilibrium phase diagrams of the studied $\mathrm{Pt}-\mathrm{M}$ alloys [8], the solubility limit of $\mathrm{V}$ in fcc $\mathrm{Pt}$ at low temperatures is about 20 at.\%, whereas that of $\mathrm{Nb}$ is about 18 at.\%, and that of $\mathrm{Ta}$ is about 15 at.\%. For this reason, we confined our numerical study to maximum content of 20 at. $\%$ of $\mathrm{M}$ dopants.

The calculated transport properties as functions of the composition are displayed in Fig. 1. One can see very similar values and concentration trends of all basic quantities: the SHC (Fig. 1a), the residual resistivity $\rho$ (Fig. 1b), and the SHA (Fig. 1c). This similarity reflects the isoelectronic nature (the same valency) of $\mathrm{V}, \mathrm{Nb}$, and $\mathrm{Ta}$, whereas their slightly different atomic sizes are of secondary importance. The most surprising feature of the obtained results is a weak sensitivity of the SHC to the strength of the impurity spin-orbit interaction (which scales quadratically with the atomic number and is thus an order of magnitude stronger for Ta than for V). A closer look at the SHC (Fig. 1a) reveals that for the dopant concentrations above 5 at.\%, all three SHC values are essentially identical. The only discernible differences can be seen for very small impurity concentrations. In the latter case, the SHC is dominated by its extrinsic part which is due to the skew-scattering
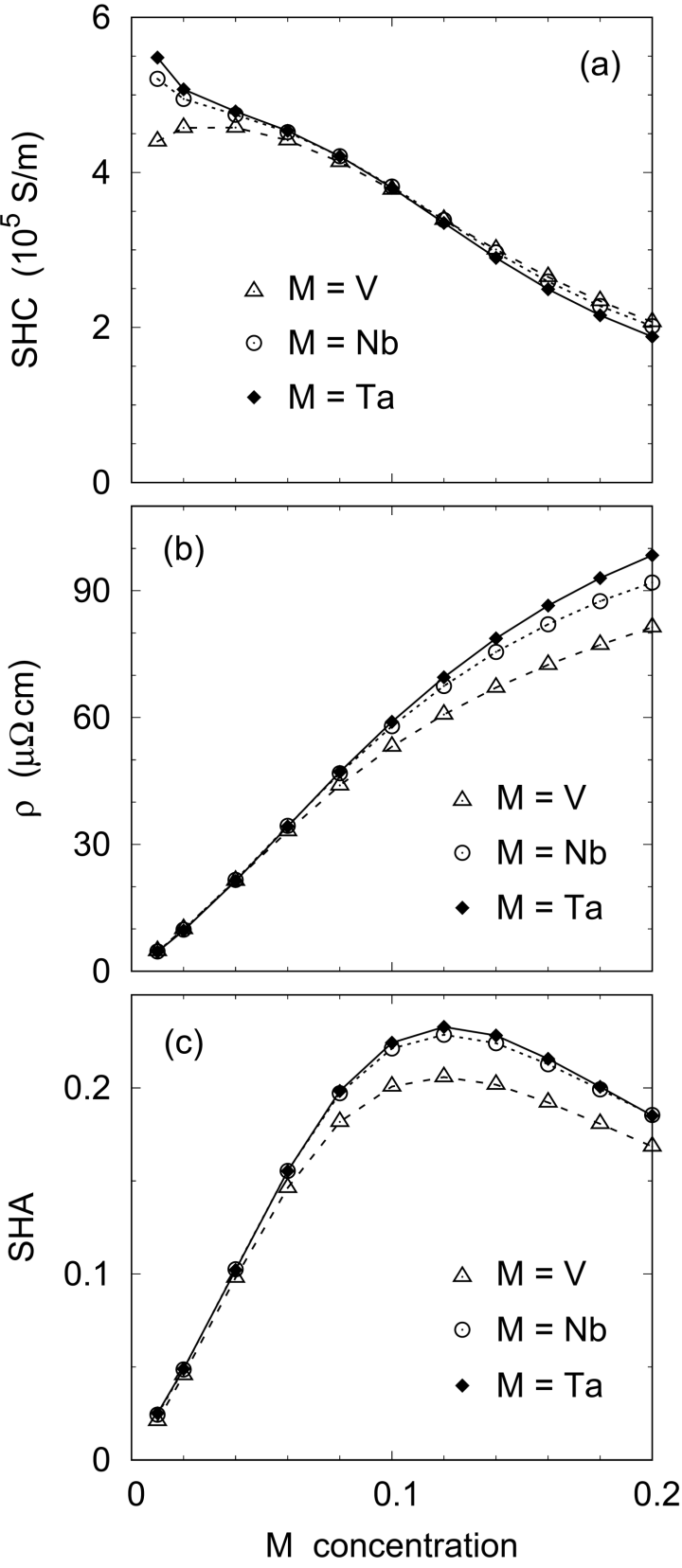

Fig. 1. Calculated transport properties of $\mathrm{Pt}-\mathrm{M}(\mathrm{M}=$ $\mathrm{V}, \mathrm{Nb}, \mathrm{Ta}$ ) alloys as functions of $\mathrm{M}$ concentration: (a) spin Hall conductivity, (b) residual resistivity $\rho$, and (c) spin Hall angle.

mechanism causing a divergence of the SHC in the dilute limit $[1,2]$. The $\mathrm{SHC}$ of the concentrated alloys is dominated by its intrinsic part, which exhibits a decrease owing to $\mathrm{M}$ alloying, in analogy to the case of random fcc $\mathrm{Pt}-\mathrm{Au}$ alloys [2].

The residual resistivity $\rho$ exhibits an increasing trend due to alloying (Fig. 1b). Thus, a consequence of the opposite trends of the SHC and $\rho$ is a maximum in the SHA encountered for $\mathrm{M}$ content around 12 at.\% (Fig. 1c). 
The maximum SHA values fall slightly above 0.2 which is comparable with the top values obtained for other systems investigated so far in this context [1-3]. The SHA maximum in the $\mathrm{Pt}-\mathrm{V}$ alloy is slightly smaller than the maximum in the $\mathrm{Pt}-\mathrm{Nb}$ and $\mathrm{Pt}-\mathrm{Ta}$ alloys. However, this difference is not due to different SHC values but rather due to the slightly different resistivities $\rho$. This fact indicates that the strength of spin-orbit interaction of the dopant element does not represent the key factor for obtaining sizable SHA and that $\rho$ plays a more significant role. It should be noted that the important effect of the longitudinal resistivity on the resulting SHA values has also been emphasized in recent studies of amorphous $\mathrm{Hf}-\mathrm{W}$ alloys [3] and of multilayers with $\mathrm{Pt}-\mathrm{Al}$ and $\mathrm{Pt}-\mathrm{Hf}$ alloys [4].

\section{Conclusions}

The performed theoretical study of the spin Hall effect in concentrated random $\mathrm{Pt}-\mathrm{V}, \mathrm{Pt}-\mathrm{Nb}$, and $\mathrm{Pt}-\mathrm{Ta}$ alloys predicts sizable values of their spin Hall angle, with a maximum found for compositions around 12 at.\% of impurities. The similar values and concentration trends of all studied transport properties indicate that the strength of impurity spin-orbit coupling plays a secondary role. A more important factor seems to be the residual resistivity of the alloys. The maximum spin Hall angles were obtained for compositions that belong to thermodynamically stable phases (primary solid solutions with an $f c c$ structure). The studied alloys might be thus viewed as alternatives to other systems considered in this context, which are often metastable crystalline or amorphous alloys, stabilized only by kinetic barriers.

\section{Acknowledgments}

The authors acknowledge financial support from the Czech Science Foundation (grant No. 18-07172S).

\section{References}

[1] J. Sinova, S.O. Valenzuela, J. Wunderlich, C.H. Back, T. Jungwirth, Rev. Mod. Phys. 87, 1213 (2015).

[2] M. Obstbaum, M. Decker, A.K. Greitner et al., Phys. Rev. Lett. 117, 167204 (2016).

[3] K. Fritz, S. Wimmer, H. Ebert, M. Meinert, Phys. Rev. B 98, 094433 (2018).

[4] M.H. Nguyen, M. Zhao, D.C. Ralph, R.A. Buhrman, Appl. Phys. Lett. 108, 242407 (2016).

[5] V. Drchal, J. Kudrnovský, P. Weinberger, Phys. Rev. $B$ 50, 7903 (1994).

[6] I. Turek, J. Kudrnovský, V. Drchal, Phys. Rev. B 89, 064405 (2014).

[7] I. Turek, J. Kudrnovský, V. Drchal, Phys. Rev. B 86 014405 (2012).

[8] Binary Alloy Phase Diagrams, Ed. T.B. Massalski, American Society for Metals, Metals Park (OH) 1986. 